\title{
Changes of End of Life Practices for Cancer Patients and Their Association with Hospice Palliative Care Referral over 2009-2014: A Single Institution Study
}

\author{
Hyun Jung Jho, MD \\ Eun Jung Nam, RN ${ }^{1}$ \\ II Won Shin, RN2 \\ Sun Young Kim, $\mathrm{MD}^{3}$
}

${ }^{1}$ Department of Hospice Palliative Service, National Cancer Center, Goyang, Information Technology Team,

National Cancer Center, Goyang,

${ }^{3}$ Department of Oncology, University of Ulsan College of Medicine, Seoul, Korea
Correspondence: Sun Young Kim, MD Department of Oncology, University of Ulsan College of Medicine, 88 Olympic-ro 43-gil, Songpa-gu, Seoul 05505, Korea

Tel: 82-2-3010-3204

Fax: 82-2-3010-6961

E-mail: sunyoungkim@amc.seoul.kr

Received November 23, 2018

Accepted September 3, 2019

Published Online September 3, 2019

\begin{abstract}
Purpose
In Korea, hospice palliative care (HPC) provision for cancer patients has increased recently. However, whether end of life (EoL) care practices have improved along with the development of HPC is unclear. We intended to investigate the changes in EoL care practices and their association with HPC referral.
\end{abstract}

\section{Materials and Methods}

Retrospective medical record review of adult cancer patients who died at National Cancer Center Korea from 1 January 2009 to 31 December 2014 was performed. Changes of EoL practices including chemotherapy within 2 weeks from death, death in intensive care unit (ICU), documentation of "do not resuscitate (DNR)" within 7 days from death and referral to HPC from 2009 to 2014 were analyzed as well as the association between referral to HPC and other practices.

\section{Results}

A total of 2,377 cases were included in the analysis. Between 2009 and 2014, referral to HPC increased and DNR documentation within 7 days from death decreased significantly. Cases for chemotherapy within 2 weeks from death and death in ICU didn't change over the study period. Patients referred to HPC were less likely to receive chemotherapy within 2 weeks from death, die in ICU and document DNR within 7 days from death.

\section{Conclusion}

During the study period, EoL practices among cancer patients partly changed toward less aggressive in our institution. HPC referral was associated with less aggressive cancer care at the EoL. Policies to promote EoL discussion are necessary to improve the EoL practices of cancer patients.

\section{Introduction}

Hospice palliative care (HPC) has drawn increasing attention throughout the globe over the last decades [1,2]. Ever since the modern hospice movement sprouted from the care for terminal cancer patients at 1960s, HPC has been closely linked with quality cancer care.

Along with the growth of HPC, cancer care at the end of life (EoL) including the aggressiveness cancer treatment and

\section{Key words}

Neoplasms, Hospice and palliative care, Terminal care, Chemotherapy, Aggressiveness 
EoL discussion is an essential component of HPC and it has been studied that EoL discussion could contribute to reduce the aggressiveness of cancer care near death $[8,9]$. EoL discussion between the medical professionals and patients and family members includes communication about disease status, prognosis, negotiating goal of care and care preference including documentation of do not resuscitation (DNR) [10]. Appropriately introduced EoL discussion has a positive effect such as avoiding unnecessary aggressive care near death, facilitating hospice utilization and improving quality of life for patient and family [9]. Therefore, it has been recommended to initiate these discussions earlier during the course of advanced cancer [8,11]. Still, studies showed that about half of the bereaved families felt that EoL discussion and referral to palliative care occurred too late [12,13].

In the Republic of Korea, where cancer is the leading cause of death, national policy has been supporting HPC for terminal cancer patients since early 2000s. In 2003, Ministry of Health and Welfare (MoHW) started the first pilot project for $\mathrm{HPC}$, and the project gradually expanded to subsidizing 23 HPC units in 2007 [14]. Subsequently, MoHW announced the standard for official designation of HPC units eligible for government's subsidy in 2008, after which number of designated HPC units continued to increase up to 57 units with 950 beds across Korea in 2014 from 19 units with 282 beds at 2008 [15]. The registry for these designated HPC units shows that the utilization of HPC units among cancer death also increased from 7.3\% to 13.8\% between 2008 and 2014 [15]. Eventually, National Health Insurance started coverage for designated inpatient hospice units in 2015.

In this study, we hypothesized that EoL practices for cancer patients might have been influenced by the recent expansion of HPC in Korea. Previous studies in Korea reported up to $22.5 \%$ of patients received chemotherapy near death in 2004 [16] while $46.8 \%$ to $70 \%$ of DNR documentation occurred within a week from death among terminal cancer patients $[17,18]$ by 2005 . We aimed to investigate whether EoL practices such as chemotherapy and DNR documentation near death have been changed after initiating designation and subsidizing HPC units in 2008 in a single cancer center and the association between HPC referral and these EoL practices.

\section{Materials and Methods}

\section{Study subject}

This is a retrospective medical record review of cancer patients who died at National Cancer Center Korea from 1
January 2009 to 31 December 2014. Cases were limited to the adult (age of death more than 19 years old) cancer patients with a primary site of lung, stomach, breast, colorectal, and hematological malignancy as these comprised most of the primary site among the decedents in National Cancer Center and mainly treated with chemotherapy. Patients who passed away within 2 weeks from the first visit to National Cancer Center were excluded.

Data were extracted by Information Technology Team and then were anonymized by medical record service before delivered to the authors. The study period was set as from 2009 due to the availability of the electronic medical record for HPC referral.

\section{EoL practices and data collection}

Based on the literature review, the authors selected four EoL practices available from medical records. These were whether (1) patients received chemotherapy within 2 weeks from death (2) patients died in ICU, and (3) patients documented DNR within 7 days from death, and (4) patients were referred to the palliative care team.

Chemotherapy near death has been used as an indicator of the aggressiveness of care in previous studies [3]. Admission to ICU in the last month of life was also regarded as to reflect aggressive care at the EoL situation. As the timing of EoL discussion and hospice referral is usually very late in Korea [19], ICU death was considered to reflect aggressive care more clearly than admitting to ICU in this setting. DNR within 7 days from death was chosen as it reflects late discussion of EoL between medical professionals and patients and family members.

The HPC team at National Cancer Center consists of physician and advanced nurse practitioner specialized in HPC. HPC team's roles encompassed comprehensive evaluation for patients and family, giving advice on symptom management, care planning, and introducing HPC facilities through consultation.

We collected data including patient's age of death, sex, primary site of cancer, date of death, date of last chemotherapy dose given, date of the first visit to National Cancer Center, educational status, type of health insurance, HPC referral status and date of DNR documentation. Patients with more than one site of cancer were assigned the diagnosis of last admission episode. Electronic data for DNR documentation and ICU death were fully available from 2011.

\section{Statistical analysis}

General characteristics of the patients were described in percentage and mean according to the year of death. Trends of the four EoL practices over the study period were ana- 
Table 1. General characteristics of subjects

\begin{tabular}{|c|c|c|c|c|}
\hline Characteristic & Total & 2009-2010 & 2011-2012 & 2013-2014 \\
\hline No. $(\%)$ & $2,377(100)$ & $833(35.0)$ & $769(32.4)$ & $775(32.6)$ \\
\hline Age of death (yr) & $60.3 \pm 12.3$ & $59.8 \pm 12.2$ & $60.3 \pm 12.5$ & $61.0 \pm 12.1$ \\
\hline \multicolumn{5}{|l|}{ Sex } \\
\hline Male & $1,454(61.2)$ & $523(62.8)$ & $458(59.6)$ & $473(61.0)$ \\
\hline Female & $923(38.8)$ & $310(37.2)$ & $311(40.4)$ & $302(39.0)$ \\
\hline \multicolumn{5}{|l|}{ Primary site of cancer } \\
\hline Lung & $1,298(54.6)$ & $498(59.8)$ & $425(55.3)$ & $375(48.4)$ \\
\hline Breast & $267(11.2)$ & $91(10.9)$ & $86(11.2)$ & $90(11.6)$ \\
\hline Stomach & $381(16.0)$ & $102(12.2)$ & $121(15.7)$ & $158(20.4)$ \\
\hline Colorectal & $217(9.1)$ & $76(9.1)$ & $70(9.1)$ & $71(9.2)$ \\
\hline Hematologic malignancy & $214(9.0)$ & $66(7.9)$ & $67(8.7)$ & $81(10.5)$ \\
\hline \multicolumn{5}{|l|}{ Years of education } \\
\hline$\leq 12$ & $1,707(71.8)$ & $606(72.8)$ & $550(71.5)$ & $551(71.1)$ \\
\hline$>12$ & $603(25.4)$ & $198(23.8)$ & $197(25.6)$ & $208(26.8)$ \\
\hline Missing & $67(2.8)$ & $29(3.5)$ & $22(2.9)$ & $16(2.1)$ \\
\hline \multicolumn{5}{|l|}{ Healthcare insurance } \\
\hline $\mathrm{NHI}$ & $2,244(94.4)$ & $784(94.1)$ & $731(95.1)$ & $729(94.1)$ \\
\hline Medical aid & $130(5.5)$ & $49(5.9)$ & $38(5.0)$ & $43(5.6)$ \\
\hline No insurance & $3(0.1)$ & 0 & 0 & $3(0.4)$ \\
\hline Received chemotherapy & $2,148(90.4)$ & $737(88.5)$ & $703(91.4)$ & $708(91.4)$ \\
\hline Last chemotherapy to death, mean \pm SD (median, day) & $68.6 \pm 126.3(33)$ & $63.3 \pm 92.3(36)$ & $70.9 \pm 151.7(31)$ & $71.8 \pm 129.2(33)$ \\
\hline DNR documentation ${ }^{\text {a) }}$ & $1,435(92.9)$ & - & $714(92.8)$ & $721(93.0)$ \\
\hline DNR documentation to death ${ }^{a}$, mean $\pm S D$ (median, day) & $7.0 \pm 15.7(3)$ & - & $6.4 \pm 13.4(3)$ & $7.7 \pm 17.7$ (3) \\
\hline
\end{tabular}

Values are presented as number $(\%)$ or mean \pm SD unless otherwise indicated. SD, standard deviation; NHI, National Health Insurance; DNR, do not resuscitate. ${ }^{\text {a) }}$ Date of DNR documentation was available from 2011 from the database.

lyzed with linear regression analysis. Association between HPC referral and the other EoL practices (chemotherapy within 2 weeks from death, death in ICU and DNR documentation within 7 days from death) was analyzed with logistic regression analysis adjusted with general characteristics. All statistical analysis was performed using STATA 12SE (Stata Corp., College Station, TX). Statistical significance was set at $\mathrm{p}<0.05$.

\section{Ethical statement}

This study was approved by the institutional review board of National Cancer Center (NCC2015-0043). Informed consent was waived as the study subjects were deceased.

\section{Results}

Three thousand seven hundred thirty cases were extracted as died from 2009 to 2014 and age of death of 20 years or older. Among these, primary sites of other than above five sites $(n=1,292)$ and who died within 2 weeks from first visit $(n=61)$ were excluded subsequently. Finally, 2,377 cases were included in the analysis.

General characteristics of study subjects are presented in Table 1. Mean age of death was $60.3 \pm 12.3$. Male patients comprised about $60 \%$ of total subjects. Lung was the most common primary site of cancer $(54.6 \%)$. About quarter of subjects had an education of college degree or higher. Five point five percentage of cases received Medical Aid program for the low-income individual.

Majority of patients $(90.4 \%)$ received chemotherapy. The median duration from last chemotherapy dose to death was 33 days. Mean duration from DNR documentation to death was $7.0 \pm 15.7$ days.

Fig. 1 describes the trend of the EoL practices between 2009 and 2014. Referral to palliative care team increased ( $p<$ 


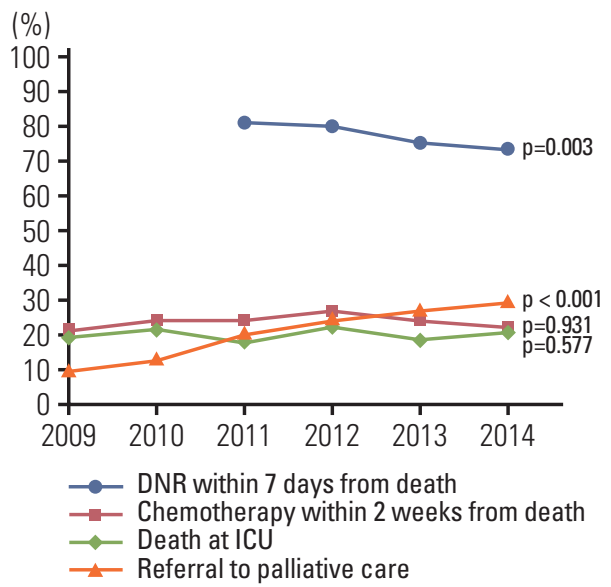

Fig. 1. Trends of end of life care practices from 2009 to 2014. DNR, Do Not Resuscitate; ICU, intensive care unit.
Table 2 presents the association between HPC referral and other EoL practices. Overall, patients referred to HPC were less likely to receive chemotherapy within 2 weeks from death, die in ICU and document DNR within 7 days from death after adjusting age, sex, primary site, educational and health insurance status.

Patients of age $>60$, stomach cancer, and colorectal cancer were less likely to receive chemotherapy within 2 weeks from death. Age $>60$, male sex, and hematologic cancer patients were associated with increased death in ICU. Hematologic cancer patients and patients with higher education level were more likely to document DNR earlier than 7 days from death.

\section{Discussion}

$0.001)$ and DNR documentation within 7 days from death decreased $(p=0.003)$ significantly, while other practices stayed unchanged over time.
We investigated the changes in EoL practices in a single cancer center in Korea during 2009-2014. We found there were positive changes in EoL practice such as increased HPC

Table 2. Association between HPC referral and EoL practices

\begin{tabular}{|c|c|c|c|c|c|c|c|c|c|}
\hline \multirow[t]{2}{*}{ Variable } & \multicolumn{3}{|c|}{$\begin{array}{l}\text { Chemotherapy within } \\
14 \text { days from death }\end{array}$} & \multicolumn{3}{|c|}{ Death in ICU } & \multicolumn{3}{|c|}{$\begin{array}{l}\text { DNR documentation } \\
\text { within } 7 \text { days from death }\end{array}$} \\
\hline & aOR & $95 \% \mathrm{CI}$ & p-value & aOR & $95 \% \mathrm{CI}$ & p-value & aOR & $95 \% \mathrm{CI}$ & p-value \\
\hline No. $(\%)$ & \multicolumn{2}{|c|}{$562(23.6)$} & & \multicolumn{2}{|c|}{$477(20.1)$} & & \multicolumn{2}{|c|}{$1,109(77.3)$} & \\
\hline HPC referral & 0.28 & $0.20-0.40$ & $<0.001$ & 0.05 & $0.02-0.11$ & $<0.001$ & 0.33 & $0.25-0.45$ & $<0.001$ \\
\hline \multicolumn{10}{|l|}{ Age (yr) } \\
\hline$\leq 60$ & 1 & & & 1 & 1 & & 1 & & \\
\hline$>60$ & 0.72 & $0.58-0.89$ & 0.003 & 1.7 & $1.35-2.15$ & $<0.001$ & 1.14 & $0.86-1.51$ & 0.366 \\
\hline \multicolumn{10}{|l|}{ Sex } \\
\hline Male & 1 & & & 1 & & & 1 & & \\
\hline Female & 1.29 & $1.02-1.63$ & 0.032 & 0.62 & $0.48-0.80$ & $<0.001$ & 0.84 & $0.62-1.13$ & 0.239 \\
\hline \multicolumn{10}{|c|}{ Primary site of cancer } \\
\hline Lung & 1 & & & 1 & & & 1 & & \\
\hline Breast & 1.03 & $0.72-1.48$ & 0.855 & 0.7 & $0.42-1.19$ & 0.192 & 0.89 & $0.56-1.43$ & 0.634 \\
\hline Stomach & 0.66 & $0.49-0.90$ & 0.008 & 0.94 & $0.68-1.30$ & 0.693 & 0.76 & 0.53-1.09 & 0.132 \\
\hline Colorectal & 0.25 & $0.13-0.46$ & $<0.001$ & 1.21 & $0.76-1.91$ & 0.427 & 0.69 & $0.44-1.07$ & 0.098 \\
\hline Hematologic & 1.21 & $0.88-1.67$ & 0.249 & 2.44 & $1.77-3.37$ & $<0.001$ & 0.34 & $0.22-0.51$ & $<0.001$ \\
\hline \multicolumn{10}{|c|}{ Years of education } \\
\hline$\leq 12$ & 1 & & & 1 & & & 1 & & \\
\hline$>12$ & 1.05 & $0.83-1.32$ & 0.687 & 1.04 & $0.81-1.34$ & 0.750 & 0.62 & $0.46-0.83$ & 0.001 \\
\hline \multicolumn{10}{|c|}{ Healthcare insurance } \\
\hline $\mathrm{NHI}$ & 1 & & & 1 & & & 1 & & \\
\hline Medical aid & 0.94 & $0.60-1.47$ & 0.773 & 0.93 & $0.54-1.57$ & 0.778 & 0.84 & $0.48-1.47$ & 0.536 \\
\hline
\end{tabular}

HPC, hospice palliative care; EoL, end of life; ICU, intensive care unit; DNR, do not resuscitate; aOR, adjusted odd ratio; CI, confidence interval; NHI, National Health Insurance. 
referral and decreased DNR documentation near death although prescription of chemotherapy near death and death in ICU didn't show a significant decrease.

The increase of referral to HPC over the study period could have a few explanations. As referral to HPC is associated with awareness and knowledge for HPC among physician, patient, and family members [13], this may reflect the improved awareness for HPC. Studies showed the increasing trend of HPC referral in Korea since the early 2000s and onward $[20,21]$ during which the national policy for promoting HPC was initiated and developed. Another potentially related factor to referral is the accessibility to HPC units. The provision of HPC has continued to grow from the early 2000s to present, and the utilization rate of designated HPC units also gradually increased during this period [15]. It was suggested that the poor accessibility became less frequently encountered as a barrier for utilizing HPC in 2014 compared with 2010 among the patients referred to the palliative care team at National Cancer Center [22], though the accessibility to $\mathrm{HPC}$ needs to be improved further.

HPC referral was associated with less chemotherapy near death, ICU death and earlier DNR documentation in this study. This is consistent with the findings from previous studies which showed that HPC referral reduced the frequency of aggressive care near death among terminal cancer patients $[8,21,23,24]$. We speculate that HPC consultation could decrease aggressive care by helping patients and family members to understand disease status and HPC through additional communication on conventional oncology practice. Additionally, referring physician might feel more prepared to discuss for less aggressive care with patients and family once patients and family members understand HPC after consultation.

The proportion of patients received chemotherapy near death didn't show significant change over the study period in our finding. Increasing trend of chemotherapy use near death was reported from 1990s to early 2000s [3,4] in the United States and Canada. In Korea, increasing trend of chemotherapy near death between 2000 and 2012 was reported [20,25], implying an association with the use of targeted chemotherapy and increased chemotherapy among older population to this trend. However, more recently, Yoo et al. [21] reported decreasing trend of chemotherapy near death among lung cancer patients during 2010-2015, signaling EoL practices might be shifting toward less aggressive direction finally. Compared with these studies, our finding could be interpreted in a few ways. It is encouraging that it didn't increase over the study period, still the proportion of chemotherapy within 2 weeks from death remained high (23.6\%). As for the chemotherapy to the very late stage of cancer, physician and patient factor have been considered as contributors for chemotherapy near death [3]. Physician may pre- scribe chemotherapy for providing hope, avoiding difficult conversation about stopping treatment or experiencing occasional case of responding to the chemotherapy at late stage [3]. Patients may ask for chemotherapy to the end for not being aware of their prognosis, having unrealistic expectation toward the effect of chemotherapy or fear of doing nothing [3]. Accordingly, it was shown that advanced EoL discussion could reduce the chemotherapy at EoL $[8,9]$. However in Korea, where both oncologists and patients suffer from lack of time for communication, it may be unrealistic to expect the EoL discussion would increase spontaneously, even with the expansion of HPC provision. Therefore, ways to enable and enhance EoL discussion between physician and patient/family are necessary. Specifically, cancer control policy needs to be designed to support not only the expansion of hospice facilities but also the settlement of proper process for cancer care, for example, insuring practical environment for EoL discussion between physician and patient to happen daily oncology practice.

Of note, distinct patterns of EoL practices were observed according to different primary sites of cancer in our result. First, patients with stomach and colorectal cancer tended to withdraw chemotherapy earlier than those with lung cancer. Usually, chemotherapeutic options including targeted agents in gastrointestinal cancers are relatively limited compared with lung or breast cancer; this might have influenced the outcome. The low rate of chemotherapy in EoL period in colorectal cancer patients was already shown in our previous study [26]. Second, hematologic malignancy was associated with more death in ICU in our result. Previous findings also suggested that patients in this group received more aggressive care and were less likely to be referred to palliative care at the EOL than patients with solid tumors $[4,27]$. Patients with hematologic malignancy could experience an unpredictable deterioration of the disease course which impedes the discussion of the transition from life-prolonging treatment to palliative care [28].

The proportion of patients for death in ICU stayed unchanged during the study period, with older patients and hematologic malignancy patients being more likely to die in ICU in this study. ICU admission among cancer patients at the EoL could be affected by comorbidity [3] and insufficiently advanced care planning. Recently, the Act on Decisions on Life-Sustaining Treatment was enforced in February 2018, which is expected to promote the discussion and documentation on patient's preference for life-sustaining treatment at dying phase, thereby avoiding futile ICU admission at the EoL.

It is meaningful DNR documentation became slightly earlier in patients' disease trajectory over the study period. The proportion of documenting DNR within 7 days from death was still as high as $77.3 \%$, similar to the previous report [18] 
On the other hand, the completion of DNR documentation was higher in this study (92.9\%) compared with 76\%-86.7\% from previous studies $[17,18]$ suggesting DNR documentation practice has been more settled for this population. Of note, earlier documentation of DNR among higher education group in our finding may reflect the difference in the degree of understanding of the terminal condition according to the level of education.

This study has a few limitations. First, data were drawn from a single institution specialized in cancer, and the pattern of EoL care practice might be different from that of other medical institutions. Therefore, caution is needed not to simply generalize the study finding. To reconcile the conflicting findings between this and other studies which investigated the change in the pattern of practice, we need multi-institutional real-world data of larger scale. Second, the study period was limited to between 2009 and 2014 due to the electronic data availability. Considering that the national hospice palliative policy was initiated and developed from early 2000s, it would have been more meaningful to include the change of practices from early 2000s to estimate the influence of the policy over time. Third, the comorbidity of patients was not included in the analysis though it could have affected ICU utilization. Lastly, other factors related to the quality EoL care such as hospice facility utilization, emergency room visit, pain control using opioid were not included in this analysis. To estimate the effect of increased number of HPC units on the practice of referral to HPC, the data for actual utilization of HPC units of the referred patients would be necessary.

Over the period 2009 to 2014, HPC referral significantly increased which was related to less aggressive care near death, and earlier documentation of DNR before death also increased among cancer patients in National Cancer Center. However, chemotherapy near death and ICU death didn't show significant decrease. Cancer control policy needs to support the process of care including communication on EoL between medical professional and patient/family. Further studies are needed to evaluate the effectiveness of HPC policy on quality cancer care.

\section{Conflicts of Interest}

Conflict of interest relevant to this article was not reported.

\section{Acknowledgments}

Authors express sincere gratitude to Dr. Mi Kyung Song Ph.D. for her valuable advice on statistical analysis of this study.

\section{References}

1. Economist Intelligence Unit. The 2015 Quality of Death Index: ranking palliative care across the world. Singapore: Lien Foundation; 2015.

2. Woitha K, Carrasco JM, Clark D, Lynch T, Garralda E, MartinMoreno JM, et al. Policy on palliative care in the WHO European region: an overview of progress since the Council of Europe's (2003) recommendation 24. Eur J Public Health. 2016;26:230-5.

3. Earle CC, Landrum MB, Souza JM, Neville BA, Weeks JC, Ayanian JZ. Aggressiveness of cancer care near the end of life: is it a quality-of-care issue? J Clin Oncol. 2008;26:3860-6.

4. Ho TH, Barbera L, Saskin R, Lu H, Neville BA, Earle CC. Trends in the aggressiveness of end of life cancer care in the universal health care system of Ontario, Canada. J Clin Oncol. 2011;29:1587-91.

5. Jang RW, Krzyzanowska MK, Zimmermann C, Taback N, Alibhai SM. Palliative care and the aggressiveness of end-oflife care in patients with advanced pancreatic cancer. J Natl Cancer Inst. 2015;107:dju424.

6. Taberner Bonastre P, Taberner Bonastre MT, Soler Company E, Perez-Serrano Lainosa MD. Chemotherapy near the end of life; assessment of the clinical practise in onco-hematological in adult patients. Farm Hosp. 2016;40:14-24.

7. Chang TS, Su YC, Lee CC. Determinants for aggressive endof-life care for oral cancer patients: a population-based study in an Asian country. Medicine (Baltimore). 2015;94:e460.

8. Temel JS, Greer JA, Muzikansky A, Gallagher ER, Admane S, Jackson VA, et al. Early palliative care for patients with metastatic non-small-cell lung cancer. N Engl J Med. 2010;363: 733-42.

9. Wright AA, Zhang B, Ray A, Mack JW, Trice E, Balboni T, et al. Associations between end-of-life discussions, patient mental health, medical care near death, and caregiver bereavement adjustment. JAMA. 2008;300:1665-73.

10. Balaban RB. A physician's guide to talking about end-of-life care. J Gen Intern Med. 2000;15:195-200.

11. Smith TJ, Temin S, Alesi ER, Abernethy AP, Balboni TA, Basch EM, et al. American Society of Clinical Oncology provisional clinical opinion: the integration of palliative care into standard oncology care. J Clin Oncol. 2012;30:880-7.

12. Teno JM, Shu JE, Casarett D, Spence C, Rhodes R, Connor S. Timing of referral to hospice and quality of care: length of stay and bereaved family members' perceptions of the timing of hospice referral. J Pain Symptom Manage. 2007;34:120-5. 
13. Morita T, Akechi T, Ikenaga M, Kizawa Y, Kohara H, Mukaiyama $\mathrm{T}$, et al. Late referrals to specialized palliative care service in Japan. J Clin Oncol. 2005;23:2637-44.

14. Hospice and Palliative Care. Hospice and palliative care in Korea: facts and figures 2017. Goyang: Hospice and Palliative Care; 2019.

15. Hospice and Palliative Care [Internet]. Goyang: Hospice and Palliative Care; 2019 [cited 2019 Jul 3]. Available from: http:// hospice.cancer.go.kr.

16. Yun YH, Kwak M, Park SM, Kim S, Choi JS, Lim HY, et al. Chemotherapy use and associated factors among cancer patients near the end of life. Oncology. 2007;72:164-71.

17. Oh DY, Kim JH, Kim DW, Im SA, Kim TY, Heo DS, et al. CPR or DNR?: end-of-life decision in Korean cancer patients: a single center's experience. Support Care Cancer. 2006;14:103-8.

18. Kim DY, Lee KE, Nam EM, Lee HR, Lee KW, Kim JH, et al. Do-not-resuscitate orders for terminal patients with cancer in teaching hospitals of Korea. J Palliat Med. 2007;10:1153-8.

19. Baek YJ, Shin DW, Choi JY, Kang J, Mo HN, Kim YH, et al. Late referral to palliative care services in Korea. J Pain Symptom Manage. 2011;41:692-9.

20. Choi Y, Keam B, Kim TM, Lee SH, Kim DW, Heo DS. Cancer treatment near the end-of-life becomes more aggressive: changes in trend during 10 years at a single institute. Cancer Res Treat. 2015;47:555-63.

21. Yoo SH, Keam B, Kim M, Kim TM, Kim DW, Heo DS. The effect of hospice consultation on aggressive treatment of lung cancer. Cancer Res Treat. 2018;50:720-8.

22. Park SJ, Nam EJ, Chang YJ, Lee YJ, Jho HJ. Factors related with utilizing hospice palliative care unit among terminal cancer patients in Korea between 2010 and 2014: a single institution study. J Korean Med Sci. 2018;33:e263.

23. Amano K, Morita T, Tatara R, Katayama H, Uno T, Takagi I. Association between early palliative care referrals, inpatient hospice utilization, and aggressiveness of care at the end of life. J Palliat Med. 2015;18:270-3.

24. Maltoni M, Scarpi E, Dall'Agata M, Schiavon S, Biasini C, Codeca $\mathrm{C}$, et al. Systematic versus on-demand early palliative care: a randomised clinical trial assessing quality of care and treatment aggressiveness near the end of life. Eur J Cancer. 2016;69:110-8

25. Lee HS, Chun KH, Moon D, Yeon HK, Lee S, Lee S. Trends in receiving chemotherapy for advanced cancer patients at the end of life. BMC Palliat Care. 2015;14:4.

26. Lee SW, Jho HJ, Baek JY, Shim EK, Kim HM, Ku JY, et al. Outpatient palliative care and aggressiveness of end-of-life care in patients with metastatic colorectal cancer. Am J Hosp Palliat Care. 2018;35:166-72.

27. Hui D, Didwaniya N, Vidal M, Shin SH, Chisholm G, Roquemore J, et al. Quality of end-of-life care in patients with hematologic malignancies: a retrospective cohort study. Cancer. 2014;120:1572-8.

28. Howell DA, Shellens R, Roman E, Garry AC, Patmore R, Howard MR. Haematological malignancy: are patients appropriately referred for specialist palliative and hospice care?: a systematic review and meta-analysis of published data. Palliat Med. 2011;25:630-41. 